\title{
Highly enantioselective direct Mannich reaction of seven-membered cyclic imines dibenzo $[b, f][1,4]$ oxazepines with acetone via organocatalysis
}

\author{
You-Qing Wang*, Yuan-Yuan Ren \\ Provincial Key Laboratory of Natural Medicine and Immuno-Engineering, Henan University, Kaifeng 475004, Henan, China
}

\section{A R T I C L E I N F O}

Article history:

Received 14 August 2014

Accepted 12 September 2014

Published 20 January 2015

\section{Keywords:}

Acetone

Asymmetric catalysis

Seven-membered cyclic imine

Mannich reaction

Organocatalysis

\begin{abstract}
A B S T R A C T
Various substituted dibenzo[b,f][1,4]oxazepines as seven-membered cyclic imines underwent a highly enantioselective direct Mannich reaction with acetone when catalyzed by proline. These reactions gave a range of optically active $\beta$-carbonyl seven-membered $N$-heterocycles with excellent enantioselectivity (93\%-98\% ee). With 2-butanone as a Mannich donor, the single regioselective product was obtained with $96 \%-97 \%$ ee. The absolute configuration of the product was assigned to be $R$ by X-ray single crystal analysis of its derivative.
\end{abstract}

(C) 2015, Dalian Institute of Chemical Physics, Chinese Academy of Sciences. Published by Elsevier B.V. All rights reserved.

\section{Introduction}

Catalytic enantioselective nucleophilic addition to imines provides the most efficient method for the synthesis of $\mathrm{N}$-containing compounds with a stereogenic center at the $\alpha$-position [1]. While a variety of enolized carbonyl compounds can be used as the nucleophile, the enantioselective direct Mannich reaction offers an attractive approach to synthesize optically active $\beta$-amino-carbonyl compounds, which are very useful chiral $\mathrm{N}$-containing compounds in biologically active natural products, pharmaceuticals, and organic synthesis [2-6]. Since the pioneering work on proline-catalyzed asymmetric Mannich reaction of acyclic aldimines produced in situ from aldehydes and amines by List [7] and Barbas et al. [8] in the 2000s, much progress in the last decade has been made on the organocatalytic asymmetric direct Mannich reaction of acyclic imine [2-5]. Although cyclic imines are good electrophilic acceptors for the construction of optically active $N$-heterocycles with a $\beta$-carbonyl group, only limited six- [9-16] and five-membered $[17,18]$ cyclic imines have been employed as the substrate in catalytic asymmetric direct Mannich reactions. The extension to other novel cyclic imines, such as seven-membered cyclic imines, continues to be a topic of interest because it provides efficient access to the corresponding seven-membered $N$-heterocycles with a stereogenic center at the $\alpha$-position.

Among the seven-membered cyclic imines, the readily available dibenzo[b,f][1,4]oxazepines [19-21] play an important role in many different biologically active compounds $[22,23]$. Because these structures have an internal $\mathrm{C}=\mathrm{N}$ bond, they are potential electrophilic acceptors for enantioselective transformations. In 2011, Zhou and coworkers [24] reported

\footnotetext{
*Corresponding author. Tel/Fax: +86-371-22864665; E-mail: wyouqing@hotmail.com This work was supported by the National Natural Science Foundation of China (21002022) and the Chinese Ministry of Education (the Scientific Research Foundation for the Returned Overseas Chinese Scholars).

DOI: 10.1016/S1872-2067(14)60225-4 | http://www.sciencedirect.com/science/journal/18722067 | Chin. J. Catal., Vol. 36, No. 1, January 2015
} 
the Ir-catalyzed asymmetric hydrogenation of ketimines containing the subunits of dibenzo[ $b, f][1,4]$ oxazepines [24]. Very recently, our group successfully performed the direct Mannich reaction of aldimines dibenzo $[b, f][1,4]$ oxazepines and acetophenone derivatives catalyzed by azetidine-2-carboxylic acid $[25,26]$. As part of our interest in organocatalytic asymmetric addition to cyclic imines $[16,27]$, we report preliminary results on the enantioselective direct Mannich reaction with excellent enantioselectivity of dibenzo[b,f][1,4] oxazepines with acetone catalyzed by proline, which afforded 11-substituted-10,11-dihydrodibenzo $[b, f][1,4]$ oxazepine derivatives that are biologically important seven-membered $N$-heterocycles [28,29].

\section{Experimental}

\subsection{General methods}

All the reactions were carried out in air without special handling unless otherwise noted. Cyclic imines 1 were obtained according to our previous publication [26]. ${ }^{1} \mathrm{H}$ NMR, ${ }^{13} \mathrm{C}$ NMR, and ${ }^{19} \mathrm{~F}$ NMR spectra were recorded in $\mathrm{CDCl}_{3}$ on a $400 \mathrm{MHz}$ instrument with tetramethylsilane (TMS) as the internal standard. Enantiomeric excess (ee) was determined by HPLC analysis using a chiral column described below. Flash column chromatography was performed on silica gel (200-300 mesh). TLC analysis was performed using glass-backed plates coated with $0.2 \mathrm{~mm}$ silica. After elution, the plate was visualized under UV illumination at $254 \mathrm{~nm}$.

\subsection{Typical procedure for the catalytic asymmetric Mannich reaction}

To the mixture of imine $\mathbf{1}(0.2 \mathrm{mmol})$ and $(S)$-proline (30 mol\%, $0.06 \mathrm{mmol}$ ) in DMF (0.4 ml) was added acetone (1.0 mmol) using a micro-syringe. The reaction mixture was stirred at room temperature for some time shown. The direct purification of the reaction mixture by column chromatography on a silica gel (petroleum ether $/$ EtOAc $=40 / 1-5 / 1$ ) gave the desired Mannich product. Racemic Mannich products were obtained when the catalyst was racemic proline.

$(R)$-1-(10,11-dihydrodibenzo[b,f][1,4] oxazepin-11-yl)propan2-one (2a): $R_{f}=0.50$ (petroleum ether $/$ EtOAc $=5 / 1$ ); $98 \%$ ee, $[\alpha]^{20} \mathrm{D}=+37.4\left(c 1.0\right.$ in $\left.\mathrm{CHCl}_{3}\right) ;{ }^{1} \mathrm{H}$ NMR $\left(400 \mathrm{MHz}, \mathrm{CDCl}_{3}\right) \delta$ 7.28-7.20 (m, 1H), 7.18-7.00 (m, 4H), 6.84 (ddd, $J=8.8,7.7,1.5$ $\mathrm{Hz}, 1 \mathrm{H}), 6.68(\mathrm{td}, J=7.9,1.6 \mathrm{~Hz}, 1 \mathrm{H}), 6.53(\mathrm{dd}, J=7.9,1.5 \mathrm{~Hz}$, $1 \mathrm{H}), 4.74(\mathrm{dd}, J=9.8,3.6 \mathrm{~Hz}, 1 \mathrm{H}), 4.44(\mathrm{~s}, 1 \mathrm{H}), 3.56$ (dd, $J=18.1$, $9.8 \mathrm{~Hz}, 1 \mathrm{H}), 2.92(\mathrm{dd}, J=18.1,3.6 \mathrm{~Hz}, 1 \mathrm{H}), 2.10$ (s, 3H); ${ }^{13} \mathrm{C} \mathrm{NMR}$ $\left(100 \mathrm{MHz}, \mathrm{CDCl}_{3}\right) \delta 207.8,157.0,143.7,137.0,132.3,129.2$, 128.1, 124.6, 124.3, 121.7, 121.2, 119.1, 119.0, 53.9, 48.9, 30.5; HRMS (ESI): $m / z$ calculated for $\mathrm{C}_{16} \mathrm{H}_{16} \mathrm{NO}_{2}[\mathrm{M}+\mathrm{H}]+254.1176$, found: 254.1172; HPLC (Chiralcel AD-H column, hexane/ $i$-PrOH $=85 / 15,0.8 \mathrm{ml} / \mathrm{min}, 254 \mathrm{~nm}): t_{1}=10.5 \mathrm{~min}, t_{2}=11.8 \mathrm{~min}$ (major, $R$ ).

$(R)$-1-(8-methyl-10,11-dihydrodibenzo[ $b, f][1,4]$ oxazepin-11yl)propan-2-one (2b): $R_{f}=0.47$ (petroleum ether $/$ EtOAc $=$ 5/1); $91 \%$ ee, $[\alpha]^{20} \mathrm{D}=+40.7\left(c 1.0\right.$ in $\left.\mathrm{CHCl}_{3}\right) ;{ }^{1} \mathrm{H}$ NMR $(400$ $\left.\mathrm{MHz}, \mathrm{CDCl}_{3}\right) \delta 7.26-7.19(\mathrm{~m}, 1 \mathrm{H}), 7.17-7.08(\mathrm{~m}, 2 \mathrm{H}), 7.03(\mathrm{td}, J$
$=7.4,1.1 \mathrm{~Hz}, 1 \mathrm{H}), 6.96(\mathrm{~d}, J=8.1 \mathrm{~Hz}, 1 \mathrm{H}), 6.47(\mathrm{dd}, J=8.1,1.5$ $\mathrm{Hz}, 1 \mathrm{H}), 6.34$ (d, $J=1.2 \mathrm{~Hz}, 1 \mathrm{H}), 4.72$ (dd, $J=9.8,3.5 \mathrm{~Hz}, 1 \mathrm{H}$ ), 4.39 (s, 1H), 3.56 (dd, $J=18.1,9.8 \mathrm{~Hz}, 1 \mathrm{H}), 2.91$ (dd, $J=18.1,3.5$ $\mathrm{Hz}, 1 \mathrm{H}), 2.16$ (s, 3H), 2.10 (s, 3H); ${ }^{13} \mathrm{C}$ NMR (100 MHz, $\left.\mathrm{CDCl}_{3}\right) \delta$ 207.9, 157.3, 141.8, 136.6, 134.3, 132.5, 129.2, 128.1, 124.3, 121.5, 121.1, 119.8, 119.3, 54.0, 48.9, 30.6, 20.6; HRMS (ESI): $m / z$ calculated for $\mathrm{C}_{17} \mathrm{H}_{18} \mathrm{NO}_{2}[\mathrm{M}+\mathrm{H}]^{+}$268.1332, found: 268.1336; HPLC (Chiralcel AD-H column, hexane $/ i-\mathrm{PrOH}=$ 85/15, $0.8 \mathrm{ml} / \mathrm{min}, 254 \mathrm{~nm}$ ): $t_{1}=11.5 \mathrm{~min}$ (major, $R$ ), $t_{2}=12.3$ $\min$.

(R)-1-(8-tert-butyl-10,11-dihydrodibenzo[b,f][1,4] oxazepin11-yl)propan-2-one (2c): $R_{f}=0.34$ (petroleum ether $/$ EtOAc $=$ 10/1); $97 \%$ ee, $[\alpha]^{20} \mathrm{D}=+38.7\left(c 1.0\right.$ in $\left.\mathrm{CHCl}_{3}\right) ;{ }^{1} \mathrm{H}$ NMR $(400$ $\left.\mathrm{MHz} \mathrm{CDCl}_{3}\right) \delta 7.25-7.18(\mathrm{~m}, 1 \mathrm{H}), 7.16-7.08(\mathrm{~m}, 2 \mathrm{H}), 7.06-6.97$ (m, 2H), $6.70(\mathrm{dd}, J=8.4,2.3 \mathrm{~Hz}, 1 \mathrm{H}), 6.54(\mathrm{~d}, J=2.2 \mathrm{~Hz}, 1 \mathrm{H})$, 4.75 (dd, $J=9.7,3.5 \mathrm{~Hz}, 1 \mathrm{H}), 4.43$ (s, 1H), 3.56 (dd, $J=18.1,9.7$ $\mathrm{Hz}, 1 \mathrm{H}), 2.93$ (dd, $J=18.1,3.5 \mathrm{~Hz}, 1 \mathrm{H}), 2.10$ (s, 3H), 1.22 (s, 9H); ${ }^{13} \mathrm{C}$ NMR $\left(100 \mathrm{MHz}, \mathrm{CDCl}_{3}\right) \delta 207.9,157.3,147.8,141.7,136.2$, 132.5, 129.2, 128.1, 124.3, 121.19, 121.16, 116.3, 116.1, 53.9, 49.0, 34.1, 31.4, 30.5; HRMS (ESI): $m / z$ calculated for $\mathrm{C}_{20} \mathrm{H}_{24} \mathrm{NO}_{2}$ $[\mathrm{M}+\mathrm{H}]+310.1802$, found: 310.1818; HPLC (Chiralcel AD-H column, hexane $/ i-\mathrm{PrOH}=85 / 15,0.8 \mathrm{ml} / \mathrm{min}, 254 \mathrm{~nm}): t_{1}=7.0$ $\min \left(\right.$ major, $R$ ), $t_{2}=7.7 \mathrm{~min}$.

$(R)$-1-(8-chloro-10,11-dihydrodibenzo[b,f][1,4] oxazepin-11yl)propan-2-one (2d): $R_{f}=0.56$ (petroleum ether $/$ EtOAc $=$ 5/1); $98 \%$ ee, $[\alpha]^{20}{ }_{\mathrm{D}}=+39.2\left(c 1.0\right.$ in $\left.\mathrm{CHCl}_{3}\right) ;{ }^{1} \mathrm{H} \mathrm{NMR}(400 \mathrm{MHz}$, $\left.\mathrm{CDCl}_{3}\right) \delta=7.31-7.19(\mathrm{~m}, 1 \mathrm{H}), 7.18-6.93(\mathrm{~m}, 4 \mathrm{H}), 6.59$ (dd, $J=$ 8.5, 2.4 Hz, 1H), 6.51-6.43 (m, 1H), $4.72(\mathrm{dd}, J=9.6,3.2 \mathrm{~Hz}, 1 \mathrm{H})$, 4.56 (s, 1H), 3.59 (dd, $J=18.2,9.8 \mathrm{~Hz}, 1 \mathrm{H}), 2.93(\mathrm{dd}, J=18.2,3.2$ $\mathrm{Hz}, 1 \mathrm{H}), 2.14$ (s, 3H); ${ }^{13} \mathrm{C}$ NMR $\left(100 \mathrm{MHz}, \mathrm{CDCl}_{3}\right) \delta 207.6,156.9$, 142.1, 138.3, 132.2, 129.6, 129.5, 128.1, 124.7, 122.8, 121.1, 118.5, 117.9, 53.7, 49.0, 30.5; HRMS (ESI): $m / z$ calculated for $\mathrm{C}_{16} \mathrm{H}_{15} \mathrm{ClNO}_{2}[\mathrm{M}+\mathrm{H}]+288.0786$, found: 288.0789; HPLC (Chiralcel $\mathrm{AD}-\mathrm{H}$ column, hexane $/ \mathrm{i}-\mathrm{PrOH}=85 / 15,0.8 \mathrm{ml} / \mathrm{min}, 254$ $\mathrm{nm}): t_{1}=13.1 \mathrm{~min}$ (major, $R$ ), $t_{2}=14.5 \mathrm{~min}$.

(R)-1-(8-fluoro-10,11-dihydrodibenzo[b,f][1,4] oxazepin-11yl)propan-2-one (2e): $R_{f}=0.45$ (petroleum ether $/$ EtOAc $=$ 5/1); $98 \%$ ee, $[\alpha]^{20} \mathrm{D}=+62.6\left(c 1.0\right.$ in $\left.\mathrm{CHCl}_{3}\right) ;{ }^{1} \mathrm{H} \mathrm{NMR}(400 \mathrm{MHz}$, $\left.\mathrm{CDCl}_{3}\right) \delta 7.28-7.23(\mathrm{~m}, 1 \mathrm{H}), 7.18-7.11(\mathrm{~m}, 2 \mathrm{H}), 7.06(\mathrm{td}, J=7.4$, $1.2 \mathrm{~Hz}, 1 \mathrm{H}$ ), 7.00 (dd, $J=8.8,5.6 \mathrm{~Hz}, 1 \mathrm{H}), 6.31$ (ddd, $J=8.7,7.7$, $2.9 \mathrm{~Hz}, 1 \mathrm{H}$ ), 6.22 (dd, $J=10.2,2.9 \mathrm{~Hz}, 1 \mathrm{H}), 4.73(\mathrm{dd}, J=9.8,3.5$ $\mathrm{Hz}, 1 \mathrm{H}), 4.56$ (s, 1H), 3.61 (dd, $J=18.1,9.8 \mathrm{~Hz}, 1 \mathrm{H}), 2.93$ (dd, $J=$ 18.1, $3.5 \mathrm{~Hz}, 1 \mathrm{H}), 2.13(\mathrm{~s}, 3 \mathrm{H}) ;{ }^{13} \mathrm{C}$ NMR (100 MHz, $\left.\mathrm{CDCl}_{3}\right) \delta$ 207.7, $159.8\left(\mathrm{~d},{ }^{1} J_{\mathrm{C}-\mathrm{F}}=240.6 \mathrm{~Hz}\right), 157.3,139.7\left(\mathrm{~d},{ }^{4} J_{\mathrm{C}-\mathrm{F}}=2.4 \mathrm{~Hz}\right)$, $138.5\left(\mathrm{~d}, 3 \mathrm{~J}_{\mathrm{C}-\mathrm{F}}=10.9 \mathrm{~Hz}\right), 132.4,129.5,128.1,124.7,122.6(\mathrm{~d}$, $\left.3 \int_{\mathrm{C}-\mathrm{F}}=10.2 \mathrm{~Hz}\right), 121.1,104.8\left(\mathrm{~d},{ }^{2} J_{\mathrm{C}-\mathrm{F}}=23.3 \mathrm{~Hz}\right), 104.6\left(\mathrm{~d},{ }^{2} \mathrm{C}_{\mathrm{C}-\mathrm{F}}=\right.$ $26.4 \mathrm{~Hz}$ ), 53.6, 49.1, 30.5; ${ }^{19} \mathrm{~F}$ NMR (376 MHz, $\mathrm{CDCl}_{3}$ ) $\delta-119.0$; HRMS (ESI): $m / z$ calculated for $\mathrm{C}_{16} \mathrm{H}_{15} \mathrm{FNO}_{2}[\mathrm{M}+\mathrm{H}]^{+} 272.1081$, found: 272.1081; HPLC (Chiralcel AD-H column, hexane/ $i$-PrOH $=85 / 15,0.8 \mathrm{ml} / \mathrm{min}, 254 \mathrm{~nm}): t_{1}=11.5 \mathrm{~min}, t_{2}=12.3 \mathrm{~min}$ (major, $R$ ).

$(R)$-1-(7-methyl-10,11-dihydrodibenzo[ $b, f][1,4]$ oxazepin-11yl)propan-2-one (2f): $R_{f}=0.24$ (petroleum ether $/$ EtOAc $=$ 10/1); $96 \%$ ee, $[\alpha]^{20} \mathrm{D}=+0.5\left(c 1.0\right.$ in $\left.\mathrm{CHCl}_{3}\right) ;{ }^{1} \mathrm{H} \mathrm{NMR}(400 \mathrm{MHz}$, $\mathrm{CDCl}_{3}$ ) $\delta 7.21$ (dd, $\left.J=7.6,1.2 \mathrm{~Hz}, 1 \mathrm{H}\right), 7.17-7.09(\mathrm{~m}, 2 \mathrm{H}), 7.03$ $(\mathrm{td}, J=7.4,1.0 \mathrm{~Hz}, 1 \mathrm{H}), 6.91(\mathrm{~d}, J=1.1 \mathrm{~Hz}, 1 \mathrm{H}), 6.67(\mathrm{dd}, J=8.0$, $1.3 \mathrm{~Hz}, 1 \mathrm{H}), 6.46(\mathrm{~d}, J=8.0 \mathrm{~Hz}, 1 \mathrm{H}), 4.72(\mathrm{dd}, J=9.8,3.5 \mathrm{~Hz}, 1 \mathrm{H})$, 
$4.33(\mathrm{~s}, 1 \mathrm{H}), 3.50(\mathrm{dd}, J=18.1,9.8 \mathrm{~Hz}, 1 \mathrm{H}), 2.90(\mathrm{dd}, J=18.1,3.5$ $\mathrm{Hz}, 1 \mathrm{H}), 2.22$ (s, 3H), $2.10(\mathrm{~s}, 3 \mathrm{H}) ;{ }^{13} \mathrm{C}$ NMR (100 MHz, $\left.\mathrm{CDCl}_{3}\right) \delta$ 207.9, 157.0, 144.0, 134.2, 132.3, 129.3, 129.1, 128.2, 125.2, 124.1, 122.0, 121.2, 119.3, 54.2, 48.7, 30.5, 20.2; HRMS (ESI): $m / z$ calculated for $\mathrm{C}_{17} \mathrm{H}_{18} \mathrm{FNO}_{2}[\mathrm{M}+\mathrm{H}]^{+}$268.1332, found: 268.1325; HPLC (Chiralcel AD-H column, hexane $/ i-\mathrm{PrOH}=$ $85 / 15,0.8 \mathrm{ml} / \mathrm{min}, 254 \mathrm{~nm}$ ): $t_{1}=15.4 \mathrm{~min}$ (major, $R$ ), $t_{2}=16.3$ $\min$.

$(R)$-1-(7-chloro-10,11-dihydrodibenzo[b,f][1,4] oxazepin-11yl)propan-2-one (2g): $R_{f}=0.32$ (petroleum ether $/$ EtOAc $=$ 5/1); $97 \%$ ee, $[\alpha]^{20} \mathrm{D}=-13.1\left(c 1.0\right.$ in $\left.\mathrm{CHCl}_{3}\right) ;{ }^{1} \mathrm{H} \mathrm{NMR}(400 \mathrm{MHz}$, $\left.\mathrm{CDCl}_{3}\right) \delta 7.30-7.22(\mathrm{~m}, 1 \mathrm{H}), 7.18-7.02(\mathrm{~m}, 4 \mathrm{H}), 6.81$ (dd, $J=8.5$, $2.4 \mathrm{~Hz}, 1 \mathrm{H}), 6.45(\mathrm{~d}, J=8.5 \mathrm{~Hz}, 1 \mathrm{H}), 4.72(\mathrm{dd}, J=9.9,3.4 \mathrm{~Hz}, 1 \mathrm{H})$, 4.47 (s, 1H), 3.54 (dd, $J=18.2,9.9 \mathrm{~Hz}, 1 \mathrm{H}$ ), 2.92 (dd, $J=18.2,3.5$ $\mathrm{Hz}, 1 \mathrm{H}), 2.12$ (s, 3H); ${ }^{13} \mathrm{C} \mathrm{NMR}\left(100 \mathrm{MHz}, \mathrm{CDCl}_{3}\right) \delta 207.7,156.7$, 143.8, 135.9, 132.2, 129.4, 128.1, 124.7, 124.6, 123.0, 121.8, 121.2, 119.6, 53.8, 48.7, 30.5; HRMS (ESI): $m / z$ calculated for $\mathrm{C}_{16} \mathrm{H}_{15} \mathrm{ClNO}_{2}[\mathrm{M}+\mathrm{H}]+288.0786$, found: 288.0785; HPLC (Chiralcel IA column, hexane $/ i-\mathrm{PrOH}=85 / 15,0.8 \mathrm{ml} / \mathrm{min}, 254 \mathrm{~nm}$ ): $t_{1}$ $=10.1 \mathrm{~min}, t_{2}=11.9 \mathrm{~min}$ (major, $R$ ).

$(R)$-1-(7-fluoro-10,11-dihydrodibenzo[b,f][1,4]oxazepin-11yl)propan-2-one (2h): $R_{f}=0.23$ (petroleum ether $/$ EtOAc $=$ 10/1); $97 \%$ ee, $[\alpha]^{20} \mathrm{D}=+8.0\left(c 1.0\right.$ in $\left.\mathrm{CHCl}_{3}\right) ;{ }^{1} \mathrm{H} \mathrm{NMR}(400 \mathrm{MHz}$, $\left.\mathrm{CDCl}_{3}\right) \delta 7.24(\mathrm{t}, J=7.6 \mathrm{~Hz}, 1 \mathrm{H}), 7.18-6.99(\mathrm{~m}, 3 \mathrm{H}), 6.84(\mathrm{dd}, J=$ 9.3, 2.7 Hz, 1H), 6.59 (td, $J=8.4,2.7 \mathrm{~Hz}, 1 \mathrm{H}), 6.48$ (dd, $J=8.7,5.7$ $\mathrm{Hz}, 1 \mathrm{H}$ ), 4.73 (dd, $J=9.9,3.4 \mathrm{~Hz}, 1 \mathrm{H}), 4.35$ (s, 1H), 3.42 (dd, $J=$ $18.1,9.9 \mathrm{~Hz}, 1 \mathrm{H}$ ), 2.89 (dd, $J=18.1,3.4 \mathrm{~Hz}, 1 \mathrm{H}), 2.11(\mathrm{~s}, 3 \mathrm{H}) ;{ }^{13} \mathrm{C}$ $\operatorname{NMR}\left(100 \mathrm{MHz}, \mathrm{CDCl}_{3}\right) \delta 207.7,156.37,156.36\left(\mathrm{~d}, 1 J_{\mathrm{C}-\mathrm{F}}=239.4\right.$ $\mathrm{Hz}), 144.4\left(\mathrm{~d}, 3_{\mathrm{C}-\mathrm{F}}=10.7 \mathrm{~Hz}\right), 133.2\left(\mathrm{~d},{ }^{4} J_{\mathrm{C}-\mathrm{F}}=2.9 \mathrm{~Hz}\right), 132.0$, $129.2,128.2,124.4,121.1,119.7\left(\mathrm{~d}, 3 J_{\mathrm{C}-\mathrm{F}}=8.8 \mathrm{~Hz}\right), 111.2\left(\mathrm{~d},{ }^{2} J_{\mathrm{C}-\mathrm{F}}\right.$ $=22.1 \mathrm{~Hz}), 108.8\left(\mathrm{~d},{ }^{2} J_{\mathrm{C}-\mathrm{F}}=24.5 \mathrm{~Hz}\right), 54.1,48.6,30.5 ;{ }^{19} \mathrm{~F}$ NMR (376 $\mathrm{MHz}, \mathrm{CDCl}_{3}$ ) $\delta$-124.4; HRMS (ESI): $\mathrm{m} / z$ calculated for $\mathrm{C}_{16} \mathrm{H}_{15} \mathrm{FNO}_{2}[\mathrm{M}+\mathrm{H}]^{+}$272.1081, found: 272.1088; HPLC (Chiralcel AD-H column, hexane $/ i-\mathrm{PrOH}=85 / 15,0.8 \mathrm{ml} / \mathrm{min}, 254$ $\mathrm{nm}): t_{1}=10.8 \mathrm{~min}, t_{2}=12.4 \mathrm{~min}$ (major, $R$ ).

$(R)$-1-(6-methyl-10,11-dihydrodibenzo[ $b, f][1,4]$ oxazepin-11yl)propan-2-one (2i): $R_{f}=0.24$ (petroleum ether $/$ EtOAc $=$ 10/1); $97 \%$ ee, $[\alpha]^{20} \mathrm{D}=+28.6\left(c 1.0\right.$ in $\left.\mathrm{CHCl}_{3}\right) ;{ }^{1} \mathrm{H}$ NMR $(400$ $\left.\mathrm{MHz} \mathrm{CDCl}_{3}\right) \delta 7.28-7.15(\mathrm{~m}, 2 \mathrm{H}), 7.11(\mathrm{~d}, J=6.7 \mathrm{~Hz}, 1 \mathrm{H}), 7.03(\mathrm{t}$, $J=7.2 \mathrm{~Hz}, 1 \mathrm{H}), 6.73(\mathrm{t}, J=7.6 \mathrm{~Hz}, 1 \mathrm{H}), 6.58(\mathrm{~d}, J=7.2 \mathrm{~Hz}, 1 \mathrm{H})$, $6.39(\mathrm{~d}, J=7.8 \mathrm{~Hz}, 1 \mathrm{H}), 4.75$ (dd, $J=9.8,3.4 \mathrm{~Hz}, 1 \mathrm{H}), 4.36(\mathrm{~s}, 1 \mathrm{H})$, 3.50 (dd, $J=18.1,9.8 \mathrm{~Hz}, 1 \mathrm{H}$ ), 2.89 (dd, $J=18.1,3.4 \mathrm{~Hz}, 1 \mathrm{H}$ ), $2.42(\mathrm{~s}, 3 \mathrm{H}), 2.10(\mathrm{~s}, 3 \mathrm{H}) ;{ }^{13} \mathrm{C}$ NMR $\left(100 \mathrm{MHz}, \mathrm{CDCl}_{3}\right) \delta 207.9$, $157.0,143.0,137.4,132.6,130.6,129.0,128.2,124.2,124.1$, 121.5, 121.1, 117.0, 54.0, 49.6, 30.5, 17.2; HRMS (ESI): $m / z$ calculated for $\mathrm{C}_{17} \mathrm{H}_{18} \mathrm{NO}_{2}[\mathrm{M}+\mathrm{H}]+268.1332$, found: 268.1341; HPLC (Chiralcel AD-H column, hexane $/ i$-PrOH $=85 / 15,0.8$ $\mathrm{ml} / \mathrm{min}, 254 \mathrm{~nm}$ ): $t_{1}=8.6 \mathrm{~min}\left(\right.$ major, $R$ ), $t_{2}=10.3 \mathrm{~min}$.

$(R)$-1-(2-methyl-10,11-dihydrodibenzo[b,f][1,4] oxazepin-11yl)propan-2-one $(\mathbf{2} \mathbf{j}): R_{f}=0.29$ (petroleum ether $/$ EtOAc $=$ 10/1); $96 \%$ ee, $[\alpha]^{20} \mathrm{D}=+39.8\left(c 1.0\right.$ in $\left.\mathrm{CHCl}_{3}\right) ;{ }^{1} \mathrm{H}$ NMR $(400$ $\left.\mathrm{MHz} \mathrm{CDCl}_{3}\right) \delta 7.10-6.98(\mathrm{~m}, 3 \mathrm{H}), 6.92(\mathrm{~s}, 1 \mathrm{H}), 6.83(\mathrm{t}, J=7.4 \mathrm{~Hz}$, $1 \mathrm{H}), 6.66(\mathrm{t}, J=7.3 \mathrm{~Hz}, 1 \mathrm{H}), 6.51(\mathrm{~d}, J=7.8 \mathrm{~Hz}, 1 \mathrm{H}), 4.67(\mathrm{dd}, J=$ 9.7, 3.4 Hz, 1H), 4.43 (s, 1H), 3.55 (dd, $J=18.0,9.7 \mathrm{~Hz}, 1 \mathrm{H}), 2.92$ (dd, $J=18.0,3.4 \mathrm{~Hz}, 1 \mathrm{H}), 2.27$ (s, 3H), 2.09 (s, 3H); ${ }^{13} \mathrm{C}$ NMR $\left(100 \mathrm{MHz}, \mathrm{CDCl}_{3}\right) \delta 207.9,155.0,143.9,137.1,133.9,132.0$, 129.6, 128.6, 124.6, 121.7, 120.9, 119.1, 119.0, 54.0, 49.0, 30.6,
20.7; HRMS (ESI): $m / z$ calculated for $\mathrm{C}_{17} \mathrm{H}_{18} \mathrm{NO}_{2}[\mathrm{M}+\mathrm{H}]^{+}$ 268.1332, found: 268.1338; HPLC (Chiralcel AD-H column, hexane $/ \mathrm{i}-\mathrm{PrOH}=85 / 15,0.8 \mathrm{ml} / \mathrm{min}, 254 \mathrm{~nm}): t_{1}=9.8 \mathrm{~min}, t_{2}=$ 13.6 min (major, $R$ ).

(R)-1-(2-tert-butyl-10,11-dihydrodibenzo[b,f][1,4] oxazepin11-yl)propan-2-one (2k): $R_{f}=0.36$ (petroleum ether $/$ EtOAc $=$ 10/1); $96 \%$ ee, $[\alpha]^{20} \mathrm{D}=+34.1$ (c 1.0 in $\left.\mathrm{CHCl}_{3}\right) ;{ }^{1} \mathrm{H}$ NMR $(400$ $\left.\mathrm{MHz} \mathrm{CDCl}_{3}\right) \delta 7.24(\mathrm{dd}, J=8.5,2.4 \mathrm{~Hz}, 1 \mathrm{H}), 7.13(\mathrm{~d}, J=2.3 \mathrm{~Hz}$, $1 \mathrm{H})$, 7.10-7.01 (m, 2H), 6.88-6.74 (m, 1H), 6.71-6.61 (m, 1H), 6.52 (dd, $J=7.9,1.1 \mathrm{~Hz}, 1 \mathrm{H}$ ), 4.71 (dd, $J=9.9,3.2 \mathrm{~Hz}, 1 \mathrm{H}$ ), 4.47 (s, 1H), 3.61 (dd, $J=18.1,9.9 \mathrm{~Hz}, 1 \mathrm{H}$ ), 2.90 (dd, $J=18.1,3.3 \mathrm{~Hz}$, 1H), $2.09(\mathrm{~s}, 3 \mathrm{H}), 1.28(\mathrm{~s}, 9 \mathrm{H}) ;{ }^{13} \mathrm{C}$ NMR (100 MHz, $\left.\mathrm{CDCl}_{3}\right) \delta$ 208.0, 154.8, 147.3, 143.9, 137.1, 131.6, 126.0, 125.0, 124.6, 121.7, 120.6, 119.1, 119.0, 54.5, 49.1, 34.4, 31.5, 30.6; HRMS (ESI): $m / z$ calculated for $\mathrm{C}_{20} \mathrm{H}_{24} \mathrm{NO}_{2}[\mathrm{M}+\mathrm{H}]+310.1802$, found: 310.1812; HPLC (Chiralcel AD-H column, hexane $/ i-\mathrm{PrOH}=$ $85 / 15,0.8 \mathrm{ml} / \mathrm{min}, 254 \mathrm{~nm}$ ): $t_{1}=7.9 \mathrm{~min}, t_{2}=9.0 \mathrm{~min}$ (major, $R)$.

$(R)$-1-(2-methoxy-10,11-dihydrodibenzo[b,f][1,4]oxazepin11-yl)propan-2-one (2l): $R_{f}=0.14$ (petroleum ether $/$ EtOAc $=$ 10/1); $96 \%$ ee, $[\alpha]^{20} \mathrm{D}=+18.6$ (c 1.0 in $\left.\mathrm{CHCl}_{3}\right) ;{ }^{1} \mathrm{H}$ NMR $(400$ $\left.\mathrm{MHz}, \mathrm{CDCl}_{3}\right) \delta 7.15-7.04(\mathrm{~m}, 2 \mathrm{H}), 6.90-6.81(\mathrm{~m}, 1 \mathrm{H}), 6.76(\mathrm{dd}, J$ $=8.7,3.0 \mathrm{~Hz}, 1 \mathrm{H}), 6.72-6.63(\mathrm{~m}, 2 \mathrm{H}), 6.53(\mathrm{dd}, J=7.9,1.2 \mathrm{~Hz}$, $1 \mathrm{H}), 4.69(\mathrm{dd}, J=9.7,3.6 \mathrm{~Hz}, 1 \mathrm{H}), 4.45(\mathrm{~s}, 1 \mathrm{H}), 3.76(\mathrm{~s}, 3 \mathrm{H}), 3.61$ (dd, $J=18.0,9.7 \mathrm{~Hz}, 1 \mathrm{H}$ ), 2.96 (dd, $J=18.2,3.3 \mathrm{~Hz}, 1 \mathrm{H}$ ), 2.13 (s, $3 \mathrm{H}) ;{ }^{13} \mathrm{C}$ NMR (100 MHz, $\left.\mathrm{CDCl}_{3}\right) \delta 207.8,156.1,151.0,144.0$, 137.1, 133.3, 124.6, 121.9, 121.6, 119.0, 118.8, 113.8, 113.4, 55.7, 53.9, 48.9, 30.6; HRMS (ESI): $m / z$ calculated for $\mathrm{C}_{17} \mathrm{H}_{18} \mathrm{NO}_{3}$ $[\mathrm{M}+\mathrm{H}]^{+}$284.1281, found: 284.1289; HPLC (Chiralcel AD-H column, hexane $/ i-\mathrm{PrOH}=85 / 15,0.8 \mathrm{ml} / \mathrm{min}, 254 \mathrm{~nm}$ ): $t_{1}=13.5$ $\min , t_{2}=21.1 \mathrm{~min}$ (major, $R$ ).

$(R)$-1-(2-chloro-10,11-dihydrodibenzo[b,f][1,4] oxazepin-11yl)propan-2-one (2m): $R_{f}=0.27$ (petroleum ether $/$ EtOAc $=$ 10/1); $98 \%$ ee, $[\alpha]^{20} \mathrm{D}=+38.0\left(c 1.0\right.$ in $\left.\mathrm{CHCl}_{3}\right) ;{ }^{1} \mathrm{H}$ NMR $(400$ $\left.\mathrm{MHz}, \mathrm{CDCl}_{3}\right) \delta 7.20(\mathrm{dd}, J=8.5,2.5 \mathrm{~Hz}, 1 \mathrm{H}), 7.15-7.02(\mathrm{~m}, 3 \mathrm{H})$, 6.91-6.82 (m, 1H), $6.71(\mathrm{dd}, J=10.9,4.1 \mathrm{~Hz}, 1 \mathrm{H}), 6.55(\mathrm{~d}, J=7.9$ $\mathrm{Hz}, 1 \mathrm{H}), 4.83-4.58(\mathrm{~m}, 1 \mathrm{H}), 4.44(\mathrm{~s}, 1 \mathrm{H}), 3.52$ (dd, $J=18.2,9.8$ $\mathrm{Hz}, 1 \mathrm{H}$ ), 2.92 (dd, $J=18.2,3.5 \mathrm{~Hz}, 1 \mathrm{H}$ ), 2.13 (s, 3H); ${ }^{13} \mathrm{C}$ NMR $\left(100 \mathrm{MHz}_{\mathrm{CDCl}}\right) \delta 207.3,155.5,143.6,136.7,133.9,129.1$, 129.0, 128.0, 124.9, 122.7, 121.6, 119.5, 119.2, 53.6, 48.5, 30.5; HRMS (ESI): $m / z$ calculated for $\mathrm{C}_{16} \mathrm{H}_{15} \mathrm{ClNO}_{2}[\mathrm{M}+\mathrm{H}]^{+} 288.0786$, found: 288.0792; HPLC (Chiralcel AD-H column, hexane/ $i-\mathrm{PrOH}$ $=85 / 15,0.8 \mathrm{ml} / \mathrm{min}, 254 \mathrm{~nm}): t_{1}=10.5 \mathrm{~min}, t_{2}=20.8 \mathrm{~min}$ (major, $R$ ).

$(R)$-1-(4-methyl-10,11-dihydrodibenzo[b,f][1,4]oxazepin-11yl)propan-2-one (2n): $R_{f}=0.24$ (petroleum ether $/$ EtOAc $=$ 10/1); $98 \%$ ee, $[\alpha]^{20}{ }_{\mathrm{D}}=+79.5\left(c 1.0\right.$ in $\left.\mathrm{CHCl}_{3}\right) ;{ }^{1} \mathrm{H}$ NMR $(400$ $\left.\mathrm{MHz}, \mathrm{CDCl}_{3}\right) \delta$ 7.17-7.09 (m, 2H), 7.03-6.92 (m, 2H), $6.86(\mathrm{t}, J=$ $7.1 \mathrm{~Hz}, 1 \mathrm{H}), 6.66(\mathrm{dd}, J=10.9,4.2 \mathrm{~Hz}, 1 \mathrm{H}), 6.54(\mathrm{dd}, J=7.9,0.9$ $\mathrm{Hz}, 1 \mathrm{H}), 4.75$ (dd, $J=9.7,3.5 \mathrm{~Hz}, 1 \mathrm{H}), 4.47$ (s, 1H), 3.66 (dd, $J=$ 18.0, 9.7 Hz, 1H), 2.97 (dd, $J=18.0,3.6 \mathrm{~Hz}, 1 \mathrm{H}), 2.44(\mathrm{~s}, 3 \mathrm{H}$ ), 2.14 (s, 3H); ${ }^{13} \mathrm{C}$ NMR $\left(100 \mathrm{MHz}, \mathrm{CDCl}_{3}\right) \delta 207.9,155.4,142.9$, 137.6, 132.8, 130.8, 130.6, 125.6, 124.7, 124.2, 122.0, 118.7, 118.6, 53.7, 48.9, 30.6, 16.3; HRMS (ESI): $m / z$ calculated for $\mathrm{C}_{17} \mathrm{H}_{18} \mathrm{NO}_{2}[\mathrm{M}+\mathrm{H}]+268.1332$, found: 268.1337; HPLC (Chiralcel AD-H column, hexane $/ i-\mathrm{PrOH}=85 / 15,0.8 \mathrm{ml} / \mathrm{min}, 254 \mathrm{~nm}$ ): $t_{1}$ $=8.1 \mathrm{~min}, t_{2}=9.6 \mathrm{~min}($ major,$R)$. 
$(R)$-1-(1,3-dimethyl-10,11-dihydrodibenzo[ $b, f][1,4]$ oxazepin11-yl)propan-2-one (2o): $R_{f}=0.30$ (petroleum ether $/$ EtOAc $=$ 10/1); $93 \%$ ee, $[\alpha]^{20} \mathrm{D}=+22.3\left(c 1.0\right.$ in $\left.\mathrm{CHCl}_{3}\right) ;{ }^{1} \mathrm{H}$ NMR $(400$ $\left.\mathrm{MHz}, \mathrm{CDCl}_{3}\right) \delta 7.07(\mathrm{dd}, J=7.9,1.1 \mathrm{~Hz}, 1 \mathrm{H}), 6.90-6.81(\mathrm{~m}, 2 \mathrm{H})$, $6.77(\mathrm{~s}, 1 \mathrm{H}), 6.69-6.60(\mathrm{~m}, 1 \mathrm{H}), 6.51(\mathrm{dd}, J=7.9,1.2 \mathrm{~Hz}, 1 \mathrm{H})$, 4.97 (dd, $J=9.3,3.8 \mathrm{~Hz}, 1 \mathrm{H}), 4.48(\mathrm{~s}, 1 \mathrm{H}), 3.74$ (dd, $J=18.2,9.3$ $\mathrm{Hz}, 1 \mathrm{H}), 2.94$ (dd, $J=18.2,3.8 \mathrm{~Hz}, 1 \mathrm{H}), 2.34(\mathrm{~s}, 3 \mathrm{H}), 2.27$ (s, 3H), 2.11 (s, 3H); ${ }^{13} \mathrm{C}$ NMR (100 MHz, $\left.\mathrm{CDCl}_{3}\right) \delta 208.2,157.6,143.2$, 138.6, 137.4, 135.1, 128.5, 127.4, 124.8, 121.6, 119.7, 118.4 118.2, 49.0, 48.6, 30.7, 20.9, 19.6; HRMS (ESI): $m / z$ calculated for $\mathrm{C}_{18} \mathrm{H}_{20} \mathrm{NO}_{2}[\mathrm{M}+\mathrm{H}]+282.1489$, found: 282.1496; HPLC (Chiralcel $\mathrm{AD}-\mathrm{H}$ column, hexane $/ \mathrm{i}-\mathrm{PrOH}=85 / 15,0.8 \mathrm{ml} / \mathrm{min}, 254$ $\mathrm{nm}): t_{1}=9.5 \mathrm{~min}\left(\right.$ major, $R$ ), $t_{2}=10.6 \mathrm{~min}$.

$(R)$-1-(10,11-dihydrodibenzo[b,f][1,4] oxazepin-11-yl)butan2-one (3a): $R_{f}=0.21$ (petroleum ether $/$ EtOAc $=10 / 1$ ); $96 \%$ ee, $[\alpha]^{20} \mathrm{D}=+36.7\left(c 0.95\right.$ in $\left.\mathrm{CHCl}_{3}\right) ;{ }^{1} \mathrm{H}$ NMR $\left(400 \mathrm{MHz} \mathrm{CDCl}_{3}\right) \delta$ 7.27-7.20 (m, 1H), 7.19-7.00 (m, 4H), $6.84(\mathrm{t}, J=7.6 \mathrm{~Hz}, 1 \mathrm{H})$, $6.68(\mathrm{t}, J=7.6 \mathrm{~Hz}, 1 \mathrm{H}), 6.53(\mathrm{~d}, J=7.9 \mathrm{~Hz}, 1 \mathrm{H}), 4.76(\mathrm{dd}, J=9.8$, $3.5 \mathrm{~Hz}, 1 \mathrm{H}), 4.46(\mathrm{~s}, 1 \mathrm{H}), 3.52$ (dd, $J=17.8,9.8 \mathrm{~Hz}, 1 \mathrm{H}), 2.90$ (dd, $J=17.8,3.5 \mathrm{~Hz}, 1 \mathrm{H}), 2.36(\mathrm{q}, J=7.3 \mathrm{~Hz}, 2 \mathrm{H}), 1.00(\mathrm{t}, J=7.3 \mathrm{~Hz}$, $3 \mathrm{H}) ;{ }^{13} \mathrm{C}$ NMR (101 MHz, $\left.\mathrm{CDCl}_{3}\right) \delta 210.7,157.0,143.7,137.0$, 132.4, 129.1, 128.1, 124.6, 124.3, 121.6, 121.1, 119.1, 118.9, 54.1, 47.6, 36.5, 7.6; HRMS (ESI): $m / z$ calculated for $\mathrm{C}_{18} \mathrm{H}_{20} \mathrm{NO}_{2}$ $[\mathrm{M}+\mathrm{H}]+268.1332$, found: 268.1351; HPLC (Chiralcel AD-H column, hexane $/ i-\mathrm{PrOH}=85 / 15,0.8 \mathrm{ml} / \mathrm{min}, 254 \mathrm{~nm}): t_{1}=9.6$ $\min , t_{2}=10.8 \min ($ major,$R$ ).

$(R)$-1-(8-tert-butyl-10,11-dihydrodibenzo[ $b, f][1,4]$ oxazepin11-yl)butan-2-one (3c): $R_{f}=0.32$ (petroleum ether $/$ EtOAc $=$ $10 / 1) ; 97 \%$ ee, $[\alpha]^{20} \mathrm{D}=+27.5\left(c 0.99\right.$ in $\left.\mathrm{CHCl}_{3}\right) ;{ }^{1} \mathrm{H}$ NMR $(400$ $\mathrm{MHz}_{\mathrm{CDCl}}$ ) $\delta$ 7.26-7.19 (m, 1H), 7.17-7.09 (m, 2H), 7.07-6.97 $(\mathrm{m}, 2 \mathrm{H}), 6.70(\mathrm{dd}, J=8.4,2.1 \mathrm{~Hz}, 1 \mathrm{H}), 6.54(\mathrm{~d}, J=2.0 \mathrm{~Hz}, 1 \mathrm{H})$, 4.77 (dd, $J=9.7,3.4 \mathrm{~Hz}, 1 \mathrm{H}$ ), 4.44 (s, $1 \mathrm{H}), 3.52$ (dd, $J=17.8,9.8$ $\mathrm{Hz}, 1 \mathrm{H}), 2.91$ (dd, $J=17.8,3.6 \mathrm{~Hz}, 1 \mathrm{H}), 2.37$ (q, $J=7.3 \mathrm{~Hz}, 2 \mathrm{H}$ ), $1.22(\mathrm{~s}, 9 \mathrm{H}), 1.01(\mathrm{t}, J=7.3 \mathrm{~Hz}, 3 \mathrm{H}) ;{ }^{13} \mathrm{C} \mathrm{NMR}\left(100 \mathrm{MHz}, \mathrm{CDCl}_{3}\right) \delta$ 210.8, 157.3, 147.7, 141.7, 136.2, 132.5, 129.1, 128.1, 124.2, 121.1, 116.3, 116.1, 54.1, 47.7, 36.6, 34.1, 31.3, 7.6; HRMS (ESI): $m / z$ calculated for $\mathrm{C}_{21} \mathrm{H}_{26} \mathrm{NO}_{2} \quad[\mathrm{M}+\mathrm{H}]^{+} 324.1958$, found: 324.1963; HPLC (Chiralcel AD-H column, hexane $/ i-\mathrm{PrOH}=$ $95 / 5,0.6 \mathrm{~mL} / \mathrm{min}, 254 \mathrm{~nm}$ ): $t_{1}=14.1 \mathrm{~min}\left(\right.$ major, $R$ ), $t_{2}=15.3$ $\min$.

\subsection{Procedure for the synthesis of $\mathbf{4}$ from Mannich product $\mathbf{2 a}$}

$(R)$-1-(10-(4-bromobenzoyl)-10,11-dihydrodibenzo[b,f][1,4] oxazepin-11-yl)propan-2-one (4): To a solution of $\mathbf{2 a}(26.0 \mathrm{mg}$, $0.1 \mathrm{mmol})$ in $\mathrm{CH}_{2} \mathrm{Cl}_{2}(2.0 \mathrm{ml})$ was added $\mathrm{Et}_{3} \mathrm{~N}(20.2 \mathrm{mg}, 0.2$ mmol) and 4-bromobenzoyl chloride (32.9 $\mathrm{mg}, 0.15 \mathrm{mmol}$ ). The reaction mixture was stirred at room temperature for $12 \mathrm{~h}$, and then water was added. The mixture was extracted with $\mathrm{CH}_{2} \mathrm{Cl}_{2}$. The organic layer was washed with brine and dried over anhydrous $\mathrm{Na}_{2} \mathrm{SO}_{4}$. After removal of the solvent, the crude product was purified by flash chromatography to afford 4 as a white solid: $R_{f}=0.15$ (petroleum ether $/$ EtOAc $=5 / 1$ ); m.p. $=$ 111-112 ${ }^{\circ} \mathrm{C}$ (from hexane and dichloromethane); $98 \%$ ee, $[\alpha]^{20} \mathrm{D}=-405.8\left(c 0.82\right.$ in $\left.\mathrm{CHCl}_{3}\right) ;{ }^{1} \mathrm{H}$ NMR (400 MHz, $\left.\mathrm{CDCl}_{3}\right) \delta$ $7.40-7.11(\mathrm{~m}, 9 \mathrm{H}), 7.10-7.01(\mathrm{~m}, 1 \mathrm{H}), 6.89(\mathrm{t}, J=6.7 \mathrm{~Hz}, 1 \mathrm{H})$, 6.76-6.45 (m, 2H), 2.80-2.55 (m, 2H), 2.08 (s, 3H); ${ }^{13} \mathrm{C}$ NMR
(100 MHz, $\left.\mathrm{CDCl}_{3}\right) \delta 204.6,153.5,152.5,134.6,131.1,130.7$, 129.6, 129.2, 128.9, 127.6, 124.4, 124.4, 123.4, 121.5, 121.1, 49.8, 30.0; HRMS (ESI): $m / z$ calculated for $\mathrm{C}_{23} \mathrm{H}_{19} \mathrm{BrNO}_{3}[\mathrm{M}+\mathrm{H}]^{+}$ 436.0543, found: 436.0542; HPLC (Chiralcel OD-H column, hexane $/ \mathrm{i}-\mathrm{PrOH}=60 / 40,0.7 \mathrm{ml} / \mathrm{min}, 254 \mathrm{~nm}): t_{1}=11.1 \mathrm{~min}$ (major, $R$ ), $t_{2}=16.9 \mathrm{~min}$.

A single crystal of compound $\mathbf{4}$ was grown from its solution in dichloromethane and hexane, which was suitable for X-ray diffraction analysis [30].

\section{Results and discussion}

The optimization of the Mannich reaction was investigated with the model reaction of seven-membered cyclic imine 1a with acetone. The results are summarized in Table 1 . The reaction was initially conducted in DMSO at room temperature using $(S)$-proline as catalyst. After the full conversion of imine 1a was observed as monitored by TLC, the desired Mannich product 2 a was isolated in $74 \%$ yield with $92 \%$ ee (Table 1, entry 1). Encouraged by this good result, the effect of other solvents such as DMF, DMA, $\mathrm{MeOH}, \mathrm{THF}, \mathrm{CH}_{2} \mathrm{Cl}_{2}$, and neat condition (solvent-free) were investigated (Table 1, entries 2-7). The reaction in DMF gave the best result with a quantitative yield

Table 1

Optimization of the Mannich reaction conditions.

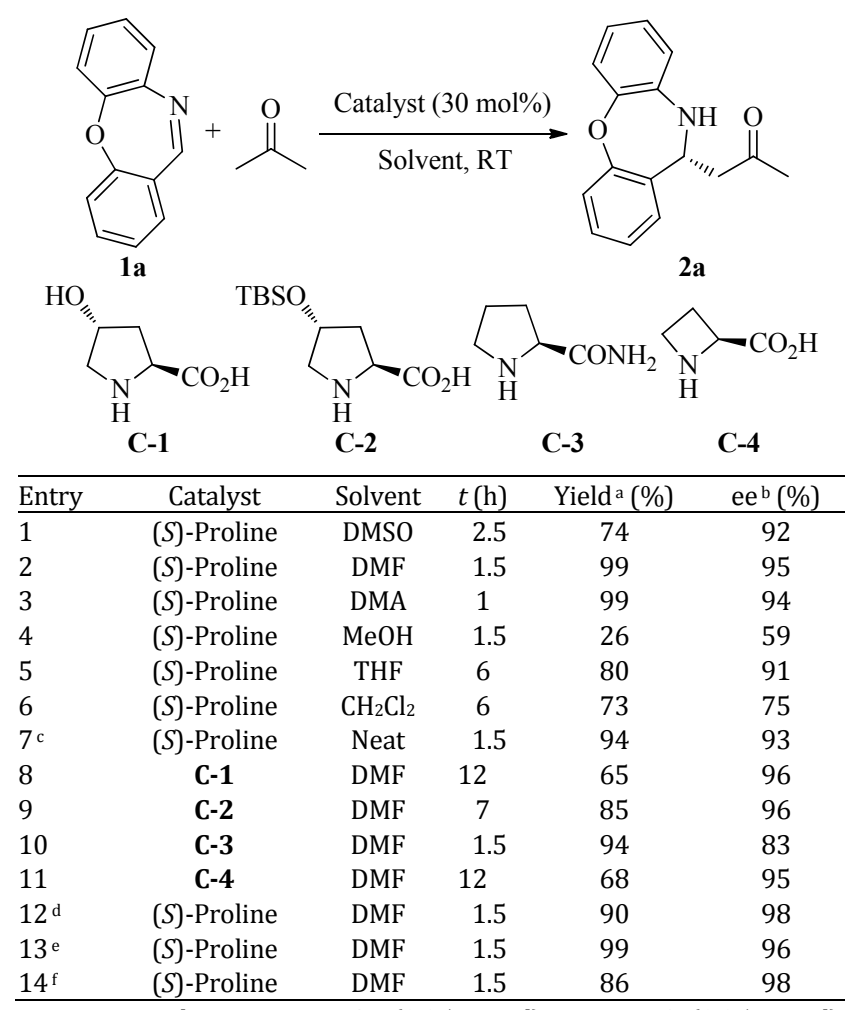

Reaction conditions: imine 1a $(0.05 \mathrm{mmol})$, acetone $2(0.25 \mathrm{mmol})$, catalysts $(0.15 \mathrm{mmol}, 30 \mathrm{~mol} \%)$, solvent $(0.1 \mathrm{ml})$.

a Isolated yield based on imine $\mathbf{1 a}$.

${ }^{\mathrm{b}}$ Determined by HPLC using a chiral column.

c $0.1 \mathrm{ml}$ of acetone was used, and no extra solvent was added.

d $10 \mathrm{mg} 3 \AA$ A MS was added.

e $10 \mathrm{mg} 4 \AA$ A MS was added.

f $10 \mathrm{mg} 5 \AA$ A MS was added. 
and excellent ee values (Table 1, entry 2). Some chiral cyclic amino acids and their derivatives were also employed as the organocatalyst to test the efficiency of this reaction (Table 1 , entries 8-11). Interestingly, in contrast to our former report on the Mannich reaction of acetophenone [26] using four-mem-

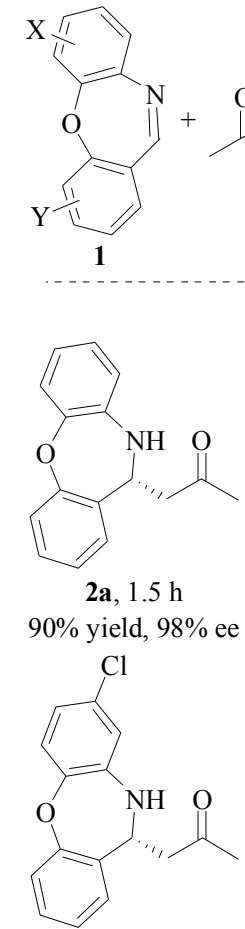

2d, $1.5 \mathrm{~h}$ $82 \%$ yield, $98 \%$ ee $\mathrm{Cl}$

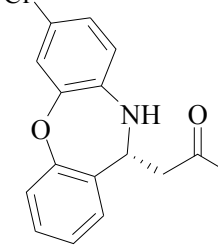

$2 \mathrm{~g}, 1.5 \mathrm{~h}$

$90 \%$ yield, $97 \%$ ee

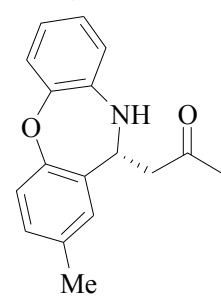

$\mathbf{2 j}, 3$ h

$96 \%$ yield, $96 \%$ ee

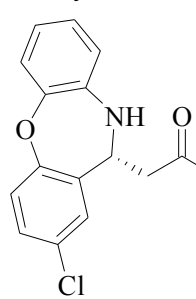

2m, 3 h

$65 \%$ yield, $98 \%$ ee
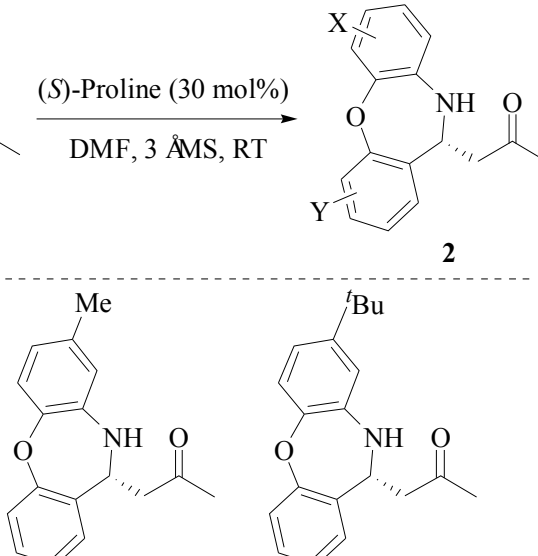

2b, $1.5 \mathrm{~h}$ 99\% yield, $91 \%$ ee

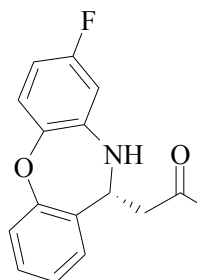

2e, $1.5 \mathrm{~h}$ $86 \%$ yield, $98 \%$ ee F

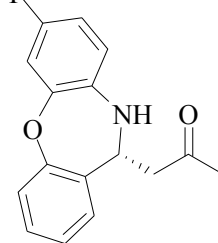

2h, 2 h

93\% yield, $97 \%$ ee

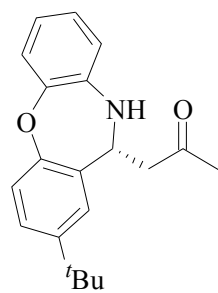

2k, 3 h

$87 \%$ yield, $96 \%$ ee

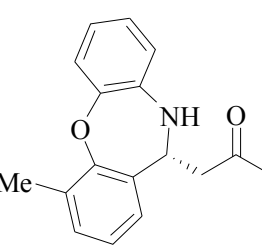

2n, 3 h $90 \%$ yield, $98 \%$ ee

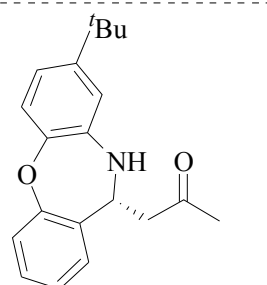

2c, $2 \mathrm{~h}$ $84 \%$ yield, $97 \%$ ee

$\mathrm{Me}$

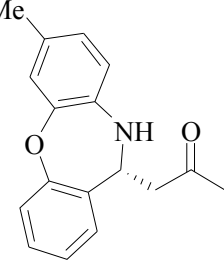

2f, $2 \mathrm{~h}$

$88 \%$ yield, $96 \%$ ee

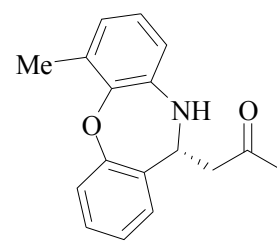

2i, 2 h

91\% yield, $97 \%$ ee

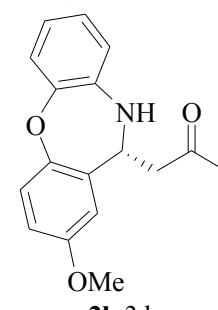

2l, $3 \mathrm{~h}$

$93 \%$ yield, $96 \%$ ee
Scheme 1. Mannich reaction of different imines 1 with acetone. bered (S)-azetidine-2-carboxylic acid (C-4) as catalyst, here the Mannich reaction with acetone afforded the same high enantioselectivity but only moderate yield (Table 1, entry 11 vs 2). Finally, the enantioselectivity was further improved to $98 \%$ ee when $3 \AA ̊$ molecular sieve (MS) was used as an additive (Table 1 , entry 12). The beneficial effect of the molecular sieve was likely due to the minimization of moisture or reducing the negative effect of the basic nitrogen atom.

Having established the optimal reaction conditions, we then examined various substituted dibenzo[b,f][1,4]oxazepines $\mathbf{1}$ and acetone to explore the generality of this enantioselective direct Mannich reaction. As listed in Scheme 1, a wide range of substituted cyclic imines $\mathbf{1}$, such as those with both electron-withdrawing and electron-donating substituents at different positions of the two aryl rings, afforded the desired Mannich products 2a-2o with excellent enantioselectivity (93\%-99\% ee) in a short time (within 3 h). For imine 1m bearing a chloro group at the para-position of the oxygen atom, the corresponding Mannich product was obtained only in moderate yield but with the same high ee value. In addition, imine 1o, which has two methyl groups on the phenyl rings, still served as a good Mannich substrate to yield 2o in high yield but with a slightly lower enantioselectivity (93\% ee) than the other imines.

With the above successful results, we further investigated the Mannich reaction of cyclic imine 1 with 2-butanone, which can form the regioisomers or diastereoisomers (Scheme 2). Under our standard conditions, the Mannich products 3a and $\mathbf{3 c}$ were isolated as the single regioisomer with excellent enantioselectivity. This indicated a high regioselectivity that favored the product resulting from the less substituted methyl of the 2-butanone.

Next, the Mannich product 2a can be smoothly converted to its $N$-4-bromobenzoyl amide 4 . The result is illustrated in Scheme 3. Treatment of $\mathbf{2 a}(98 \%$ ee) with 4-bromobenzoyl chloride led to the isolation of $\mathrm{N}$-protected product 4 in $85 \%$ yield with the same $98 \%$ ee, which was determined by HPLC analysis. Subsequently, the absolute configuration of compound 4 was established by single crystal X-ray analysis, and the stereochemistry of Mannich product 2a was assigned the $R$-configuration [30]. The absolute configurations of all the other Mannich products were assigned by analogy (Scheme 1). Based on these results, we suggest a transition state for the catalytic asymmetric Mannich reaction. The carbon nucleophile of an active enamine from acetone is much more likely to attack the seven-membered imines 1 from the re-face [26], which affords the product as $R$ (Scheme 4 ).

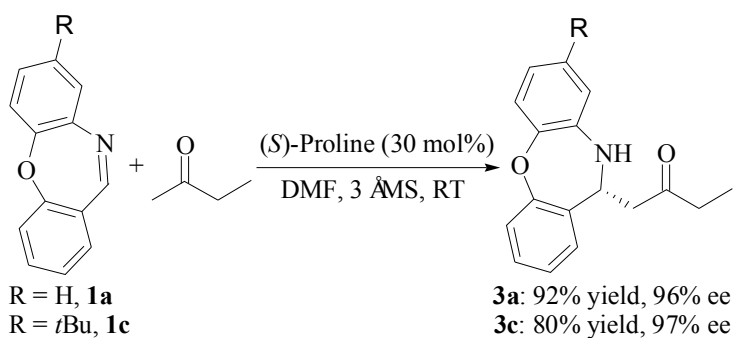

Scheme 2. Mannich reaction of cyclic imines with 2-butanone. 

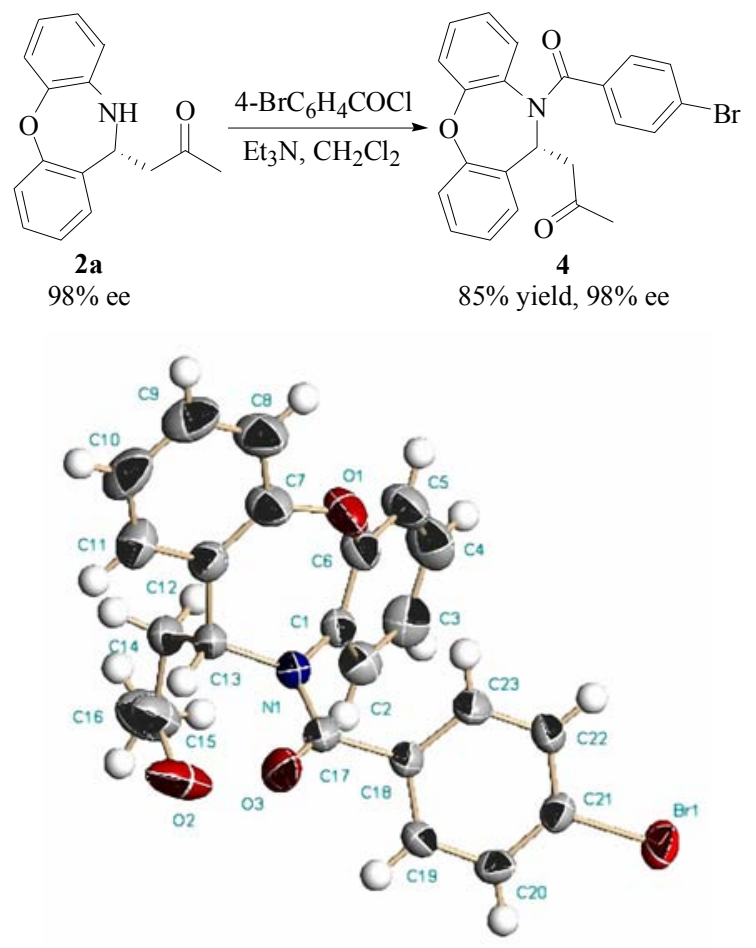

Scheme 3. Synthesis of $\mathbf{4}$ and its X-ray structure.

\section{Conclusions}

A highly enantioselective direct Mannich reaction of seven-membered cyclic imines dibenzo[b,f][1,4] oxazepines with acetone was catalyzed by proline. This provided optically active 11-substituted-10,11-dihydrodibenzo[b,f][1,4] oxazepine derivatives as the $\beta$-carbonyl seven-membered $N$-heterocycles with 93\%-98\% ee. Further studies on the Mannich reactions of dibenzo[b,f][1,4] oxazepines with other alkyl acetones will be reported later.<smiles>CC(C)CN1CCCC1C(=O)OCc1ccccc1Oc1ccccc1Cl</smiles>

Scheme 4. Transition state.

\section{References}

[1] Kobayashi S, Mori Y, Fossey J S, Salter M M. Chem Rev, 2011, 111: 2626

[2] Ting A, Schaus S E. Eur J Org Chem, 2007: 5797

[3] Verkade J M M, van Hemert L J C, Quaedflieg P J L M, Rutjes F P J T. Chem Soc Rev, 2008, 37: 29

[4] Arrayás R G, Carretero J C. Chem Soc Rev, 2009, 38: 1940

[5] Greco S J, Lacerda V Jr, dos Santos R B. Aldrichim Acta, 2011, 44: 15

[6] Karimi B, Enders D, Jafari E. Synthesis, 2013, 45: 2769

[7] List B. J Am Chem Soc, 2000, 122: 9336

[8] Notz W, Sakthivel K, Bui T, Zhong G, Barbas C F III. Tetrahedron Lett, 2001, 42: 199

[9] Itoh T, Yokoya M, Miyauchi K, Nagata K, Ohsawa A. Org Lett, 2003, 5: 4301

[10] Zhuang W, Saaby S, Jørgensen K A. Angew Chem Int Ed, 2004, 43: 4476

[11] Jiang B, Dong J J, Si Y G, Zhao X L, Huang Z G, Xu M. Adv Synth Catal, 2008, 350: 1360

[12] Hahn B T, Frőhlich R, Harms K, Glorius F. Angew Chem Int Ed, 2008, 47: 9985

[13] Schulz K, Ratjen L, Martens J. Tetrahedron, 2011, 67: 546

[14] Monaco M R, Renzi P, Schietroma D M S, Bella M. Org Lett, 2011, 13: 4546

[15] Taichi K, Song S, Kubota Y, Maruoka K. Angew Chem Int Ed, 2012, 51: 1191

[16] Wang Y Q, Zhang Y N, Pan K, You J X, Zhao J. Adv Synth Catal, 2013,

\section{Graphical Abstract}

Chin. J. Catal., 2015, 36: 93-99 doi: 10.1016/S1872-2067(14)60225-4

Highly enantioselective direct Mannich reaction of seven-membered cyclic imines dibenzo[b,f][1,4]oxazepines with acetone via organocatalysis

You-Qing Wang*, Yuan-Yuan Ren

Henan University

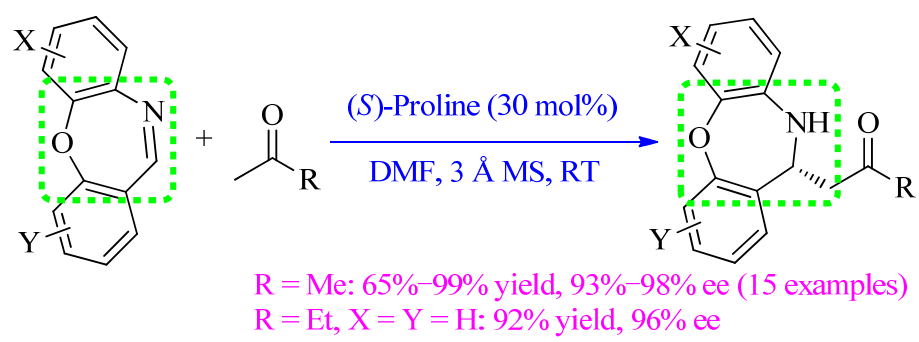

With $(S)$-proline as an organocatalyst, a direct Mannich reaction between dibenzo[b,f][1,4] oxazepines and acetone or 2-butanone afforded the optically active $\beta$-carbonyl seven-membered $N$-heterocycles with excellent enantioselectivity. 
355: 3381

[17] Li L Q, Han M Y, Xiao M X, Xie Z X. Synlett, 2011: 1727

[18] Cheng D J, Tian S K. Adv Synth Catal, 2013, 355: 1715

[19] Wardrop A W H, Sainsbury G L, Harrison J M, Inch T D. J Chem Soc, Perkin Trans 1, 1976: 1279

[20] Noskov V G, Kalinina L N, Noskova M N, Kruglyak Yu L, Strukov O G, Bezrukov A P, Kurochkin V K. Pharm Chem J, 1997, 31: 431

[21] Jorapur Y R, Rajagopal G, Saikia P J, Pal R R. Tetrahedron Lett, 2008, 49: 1495

[22] Foster R W, Weston K M. Pain, 1986, 25: 269

[23] Brône B, Peeters P J, Marrannes R, Mercken M, Nuydens R, Meert T, Gijsen H J M. Toxicol Appl Pharm, 2008, 231: 150

[24] Gao K, Yu C B, Li W, Zhou Y G, Zhang X M. Chem Commun, 2011, 47: 7845

[25] Ren Y Y, Liu S, Pan K, Wang Y Q. In: Proceedings of CCS the 8th
National Organic Chemistry Conference. Chongqing: Southwest Univ (任圆圆, 刘爽, 潘坤, 汪游清. 见: 中国化学会第八届有机化 学学术会议论文集. 重庆: 西南大学), 2013.844

[26] Ren YY, Wang Y Q, Liu S, Pan K. ChemCatChem, 2014, 6: 2985

[27] Wang Y Q, Zhang Y N, Dong H N, Zhang J, Zhao J. Eur J Org Chem, 2013, 2013: 3764

[28] Riley R J, Roberts P, Kitteringham N R, Park B K. Biochem Pharmacol, 1990, 39: 1951

[29] Dols P P M A, Folmer B J B, Hamersma H, Kuil C W, Lucas H, Ollero L, Rewinkel J B M, Hermkens P H H. Bioorg Med Chem Lett, 2008, 18: 1461

[30] CCDC 1008680 (4) contains the supplementary crystallographic data for this paper. These data can be obtained free of charge from The Cambridge Crystallographic Data Centre via www.ccdc.cam.ac.uk/data_request/cif

\title{
高对映选择性有机催化的七元环状亚胺二苯并1,4-氧氮杂卓和 丙酮的直接Mannich反应
}

\author{
汪游清", 任圆圆 \\ 河南大学天然药物与免疫工程重点实验室, 河南开封 475004
}

\begin{abstract}
摘要: 用脯氨酸作为催化剂, 研究了各种取代的二苯并1,4-氧氮杂卓衍生物类七元环状亚胺和丙酮的直接Mannich反应, 该反应能 高对映选择得到一系列旋光活性的含有 $\beta$ 羰基的七元环状氮杂环化合物( $93 \%-98 \%$ ee). 用丁酮作为Mannich给体时,能得到专一的 区域选择性和 $96 \%-97 \%$ ee的产物. 进一步通过X射线单晶衍射分析其中一个产物的衍生物, 确定了产物手性中心绝对构型为 $R$, 其 它同类型产物绝对构型随后通过化学类比方法推断确认.
\end{abstract}

关键词: 丙酮; 不对称催化; 七元环状亚胺; Mannich反应; 有机催化

收稿日期: 2014-08-14. 接受日期: 2014-09-12. 出版日期: 2015-01-20.

*通讯联系人. 电话/传真: (0371)22864665; 电子信箱: wyouqing@hotmail.com

基金来源：国家自然科学基金(21002022); 教育部留学回国人员科研启动基金.

本文的英文电子版由Elsevier出版社在ScienceDirect上出版(http://www.sciencedirect.com/science/journal/18722067). 